\title{
The SocRob Project: Soccer Robots or Society of Robots
}

\author{
Pedro U. Lima \& Luis M. M. Custodio
}

\section{Introduction}

Cooperative Robotics is a modern research field, with applications to areas such as building surveillance, transportation of large objects, air and underwater pollution monitoring, forest fire detection, transportation systems, or search and rescue after largescale disasters (Balch, T. \& Parker, L., 2002). In short, a population of cooperative robots behaves like a distributed robot to accomplish tasks that would be difficult, if not impossible, for a single robot. Many lessons important for this domain can be learned from the Multi-Agent Systems field of Artificial Intelligence (AI) concerning relevant topics for Cooperative Robotics, such as distributed continual planning (desJardins, M. E., et al, 1999), task allocation (Ferber, J., 1999), communication languages or coordination mechanisms (Decker, K. S., \& Lesser, V. R., 1995).

Robotic soccer is a very challenging problem, where the robots must cooperate not only to push and/or kick an object (a ball) towards a target region (the goal), but also to detect and avoid static (walls, stopped robots) and dynamic (moving robots) obstacles while moving towards, moving with or following the ball. Furthermore, they must cooperate to defeat an opposing team. All these are features common to many other cooperative robotics problems.

This paper surveys the several research problems addressed by the SocRob project, carried out by the Intelligent Systems Laboratory at the Institute for Systems and Robotics Instituto Superior Técnico (ISR/IST) in Lisbon, building a Systems Theory standpoint on AI concepts. In Section 2, we describe our view of the general problem involving multiple robots that act as a team, cooperating and coordinating their actions to attain the team goal. Needless to say, single-robot "traditional" research problems are covered, both from the sub-system and from the integration standpoints. Natural extensions to cooperative multi-robot teams are also detailed. The problems addressed so far and the solutions we obtained for them are described in Section 3. Open problems of interest for the project and clues on how we intend to approach their solution are discussed in Section 4 . We end the paper drawing some conclusions in Section 5.

\section{A General Multi-Robot Cooperation and Coordination Problem}

Many researchers around the world are designing mobile robots capable to display increasing autonomy and machine intelligence properties. Most groups concentrate on specific subsystems of a robot, such as the planner, the navigator, or the sensor fusion. What usually is missing in their design is a systematic way to glue together all these 
subsystems in a consistent fashion. Such a methodology, should one be available, would help engineering the mobile robots of the future.

One of the key factors of success for a robot lies on its capability to perceive correctly its surrounding environment, and to build models of the environment adequate for the task the robot is in charge of, from the information provided by its sensors. Different sensors (e.g., vision, laser, sonar, encoders) can provide alternative or complementary information about the same object, or information about different objects. Sensor fusion is the usual designation for methods of different types to merge the data from the several sensors available and provide improved information about the environment (e.g., about the geometry, color, shape and relevance of its objects).

When a team composed of several cooperating robots is concerned, the sensors are spread over the different robots, with the important advantage that the robots can move (thus moving its sensors) to actively improve the cooperative perception of the environment by the team. The information about the environment so obtained can be made available and regularly updated by different means (e.g., memory sharing, message passing, using wireless communications) to all the team robots, so as to be used by each robot subsystems. Once the information about the world is available, one may think of using it to make the team behave autonomously and machine-wise intelligently.

Three main questions arise for the team:

- Where and which a priori knowledge about the environment, team, tasks and goals, and perceptual information gathered from sensors, should be kept, updated and maintained? This involves the issue of distributed knowledge representation adequate to consistently handle different and even opposite views of the world.

- What must be done to achieve a given goal, given the constraints on time, available resources and distinct skills of the team robots? The answer to this should provide a team plan.

- How is the actual execution of a plan handled, ensuring the consistency of individual and team (sub)-goals?

So far, a bottom-up approach to the implementation of a cooperative multi-robot team has been followed in the SocRob project, starting from the development of single robot subsystems (e.g., perception, navigation, decision-making) and moving towards relational behaviors, comprehending more than one robot.

However, a key point is a top-down approach to system design. The design phase establishes the specifications for the system:

- qualitative specifications - concerning formal logical task design so as to avoid deadlocks, livelocks, unbounded resource usage and/or sharing non-sharable resources, and to choose the primitive tasks that will span the desired task space;

- quantitative specifications - concerning performance features, such as accuracy (e.g., the spatial and temporal resolution, as well as the tolerance interval around the goal, at each abstraction level), reliability and/or minimization of task execution time given a maximum allowed cost.

To support this top-down design and bottom-up implementation philosophy, suitable functional and software architectures, respectively, must be conceived prior to the development of all the sub-systems. 


\subsection{Single-Robot Research Problems}

Most of the problems tackled so far within the SocRob project concern the sub-systems of the individual robots composing a team. From our standpoint, relevant topics are:

Functional and Software Architectures: Modern robots should be designed based on a top-down design from specifications to ensure desired performance levels (both qualitative and quantitative). Therefore, the designers should start by specifying a functional architecture which will guide the design of the robot sub-systems in an integrated fashion, i.e., each sub-system is not necessarily designed to optimize its performance but rather aiming at optimizing the overall system performance. Another important issue is to determine, given the desired task space (i.e., the set of tasks that will have to be carried out by the robot in a particular application), the minimal set of primitive tasks that will span that task space. Moreover, the final implementation should be supported on a suitable software architecture designed to allow real-time multiprocessing, information sharing and mutually exclusive allocation of shared resources among the robot sub-systems.

Single-Robot Task Planning: Given the primitive task set referred in the previous item, the robot must be able, given the current and past world states (including its own internal state), to compose primitive tasks so as to come up with a plan that carries out a given desired task. There may be more than one plan that accomplishes a task, but a posterior decision system should be able to determine, eventually based on machine learning, the one that achieves the best performance, based on the available information and prediction horizon.

Single-Robot Task Coordination: Plans must be such that they allow continuous handling of the environment uncertainties and unexpected events. Once a plan is determined, task coordination deals with its execution. Plan execution must, at least, take into account the detection of events, smooth transitions between primitive tasks, synchronization of primitive tasks executed concurrently, mutual exclusion when two or more tasks attempt to access shared resources, iterative estimation of primitive task performance, learning how to improve a plan over time by choosing more convenient algorithms among those available for each primitive task, and so on.

Navigation: The navigation system is an important sub-system of a mobile robot. In many applications one important feature of the navigation system concerns the ability of the robot to self-localize, i.e., to autonomously determine its position and orientation (posture). Using posture estimates, the robot can move towards a desired posture, i.e., by following a pre-planned virtual path or by stabilizing its posture smoothly (Canudas de Wit, C., et al, 1996). If the robot is part of a cooperative multi-robot team, it can also exchange the posture information with its teammates so that appropriate relational and organizational behaviors may be established. In robotic soccer, these are crucial issues. If a robot knows its posture, it can move towards a desired posture (e.g., facing the goal with the ball in between). It can also know its teammate postures and prepare a pass, or evaluate the game state from the team robot locations. Most approaches to navigation determine with high accuracy the posture of the robot with respect to a given coordinate frame. However, this approach is typically resource-consuming, requiring a robot to spend a significant percentage of its processing time with the navigation sub-system, 
disregarding other important sub-systems, such as perception or planning, to name but a few. Furthermore, high accuracy is not always required for navigation purposes. One may be just interested to move closer to an object, rotate to see a given landmark, or move to another region. In those cases, another approach to navigation, known as topological (or relative) navigation, is advisable.

Object Recognition and Tracking Using Sensor Fusion: The ability to discriminate and recognize its surrounding objects, to distinguish the relevant ones and to track them, are major problems for any robot. For soccer robots, this problem is simplified since the relevant objects are distinguished by their colors (e.g., the ball is orange, the goals are blue and yellow). Nevertheless, fast and reliable color segmentation is not a trivial problem and requires some attention too. Furthermore, object detection may be performed by more than one sensor, such as different virtual sensors based on the vision transducer (e.g., mass center, edge detector, color segmentation), sonars, infrared and others. Therefore, sensor fusion arises as an important topic.

\subsection{Cooperative Multi-Robot Research Problems}

Functional and Software Architectures: If a team of cooperative robots is involved, the single-robot architectures of each of the team members must be integrated in the overall team architecture. The most usual solutions concerning the software architecture are - centralized, where one of the robots (or an external machine) processes the data acquired and sent by all the team members, takes all the team decisions and sends commands to the others;

- distributed, where local data processing is made at each of the robots but then information is sent to one of them to take the decisions;

- fully decentralized, where each robot takes its own decisions based on its own data and on information exchanged with its teammates.

The functional architecture of a behaviour-based multi-robot team must also classify behaviours according to the distribution of responsibilities by the team robots. One such division consists of considering organizational, relational and individual behaviours (Drogoul, A., and Collinot, A., 1998), further described below.

Multi-Robot Task Planning and Allocation: In the multiple-robot case, plans must take into account the distributed nature of the task at hand. Different tasks must be allocated to the different robots in the team, according to their skills and performance. So, the planning and task allocation system must be able to establish (sub)groups of robots within a team, and the robots must have and know how to deal with the notion of "belonging to a group". Therefore, plans must also include synchronization and communication among team members involved in the task. Moreover, if a robot cannot fulfill its assigned task, the task may simply be re-assigned to a robot within the group, a new robot may be integrated in the group to perform that task, or in the worst case a re-planning strategy has to be applied.

Multi-Robot Task Coordination: The extension of task coordination to a team of multiple robots introduces issues related to knowledge distribution and maintenance, as well as communications and related problems (e.g., noise, protocols, limited bandwidth). Furthermore, communication can be explicit (e.g., through wireless radio-frequency channels) or implicit (e.g., through the observation of teammate actions, should an a priori 
model of the teammates behaviour exist). The coordination of a task carried out by a team of cooperating robots involves signalling events detected by one robot which are relevant for some or all of its teammates and/or to exchange information obtained locally by the different robots of the team. Whenever a formation is required, several formation topologies are possible and the one suitable for the task at hand must be chosen as part of the coordination process. Although not inevitable, communications among team members are also required to keep the formation under control.

When the population is composed of heterogeneous robots, if a robot has to perform a particular task for which it does not have the necessary skills, it may ask another robot with the adequate skills to carry it out. In the particular case of the SocRob robotic team, where the robots are homogeneous, examples of cooperative behaviour are the cooperative localization of the ball, the execution of a pass, the dynamical exchange of player roles or the decision of which robot should go for the ball. All of them require some form of inter-robot coordination and underlying teamwork methodologies.

Distributed World Modeling: A team composed of multiple robots, possibly heterogeneous concerning on board sensing, can benefit from the availability of a world model, obtained from the observations made by the different team members and its on board sensors. This world model can be richer than if it were obtained by a single robot, due to the coverage of a broader area by a more diversified set of sensors. It can also be distributed through the teammates, e.g., by keeping in a robot information which is only relevant locally and by broadcasting information gathered locally but which is of interest for the team as a whole. The sensor fusion problem is similar to the single-robot case, with the important difference that the sensor subsets are now independently mobile and can be actively positioned to improve the determination of object characteristics.

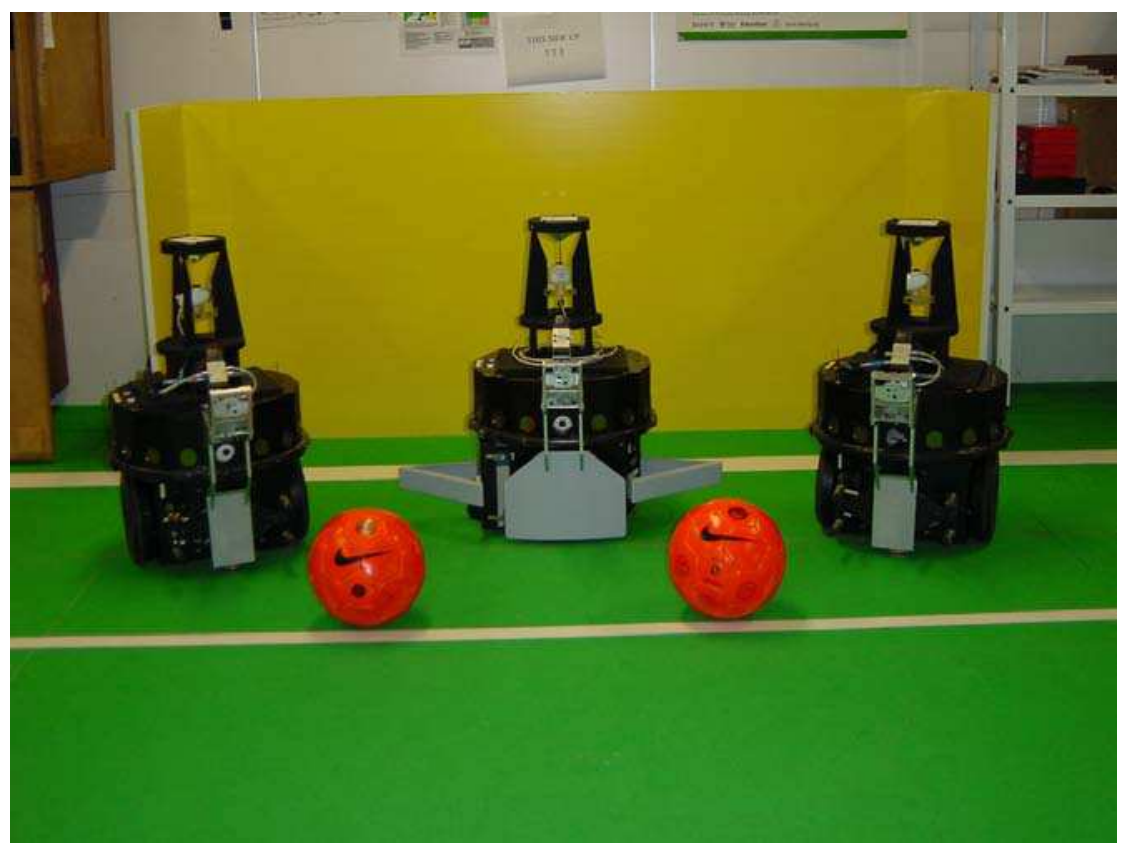

Figure 1. Three robots of the current SocRob team

\section{Problems Already Addressed}

A key issue of the research work developed under the SocRob project is the application of conceptual results to real robots participating in the Middle Size League (MSL) of RoboCup. The current robot team, displayed in Fig. 1, is composed of 4 Nomadic Super 
Scout II commercial platforms, later significantly modified by our group, each of them including:

- Two-wheel differential drive kinematics;

- Sixteen sonar sensors radially distributed around the robot, equally spaced;

- Motorola MC68332 based daughter board with three-axis motor controller, sonar and bumper interface, and battery level meters;

- Two 12V batteries, 18Ah capacity;

- Pentium III 1000MHz based motherboard, with 512MB RAM, 8GB disk;

- Two Philips USB WebCam 740K Pro. One of the WebCams looks ahead of the robot (front cam), while the other, together with a convex mirror, designed to directly obtain the soccer field bird's eye view, preserves the field geometry in the image (up cam);

- IEEE 802.11b wireless Ethernet PCMCIA card;

- Pneumatic kicking device, based on Festo components, plus one bottle for pressurized air storage;

In the remaining subsections, we describe some of the research problems addressed and solved for this team of robots.

\subsection{Color Segmentation and Cooperative Object Recognition}

A color segmentation interface was developed, providing two alternatives to discriminate the relevant MSL colors in HSV (Hue-Saturation-Value) color space (Gonzalez, R., \& Woods, R., 1992): i) adjusting HSV intervals or ii) graphically selecting regions with a given pixel color. The two approaches are cumulative. Furthermore, object segmentation is a topic directly related to the previous one, as we discriminate objects, namely the ball and the goals, not only based on their color, but also on their shape (e.g., by fitting circles to observed orange bulbs and identifying the ball with the closest and more circular bulb).

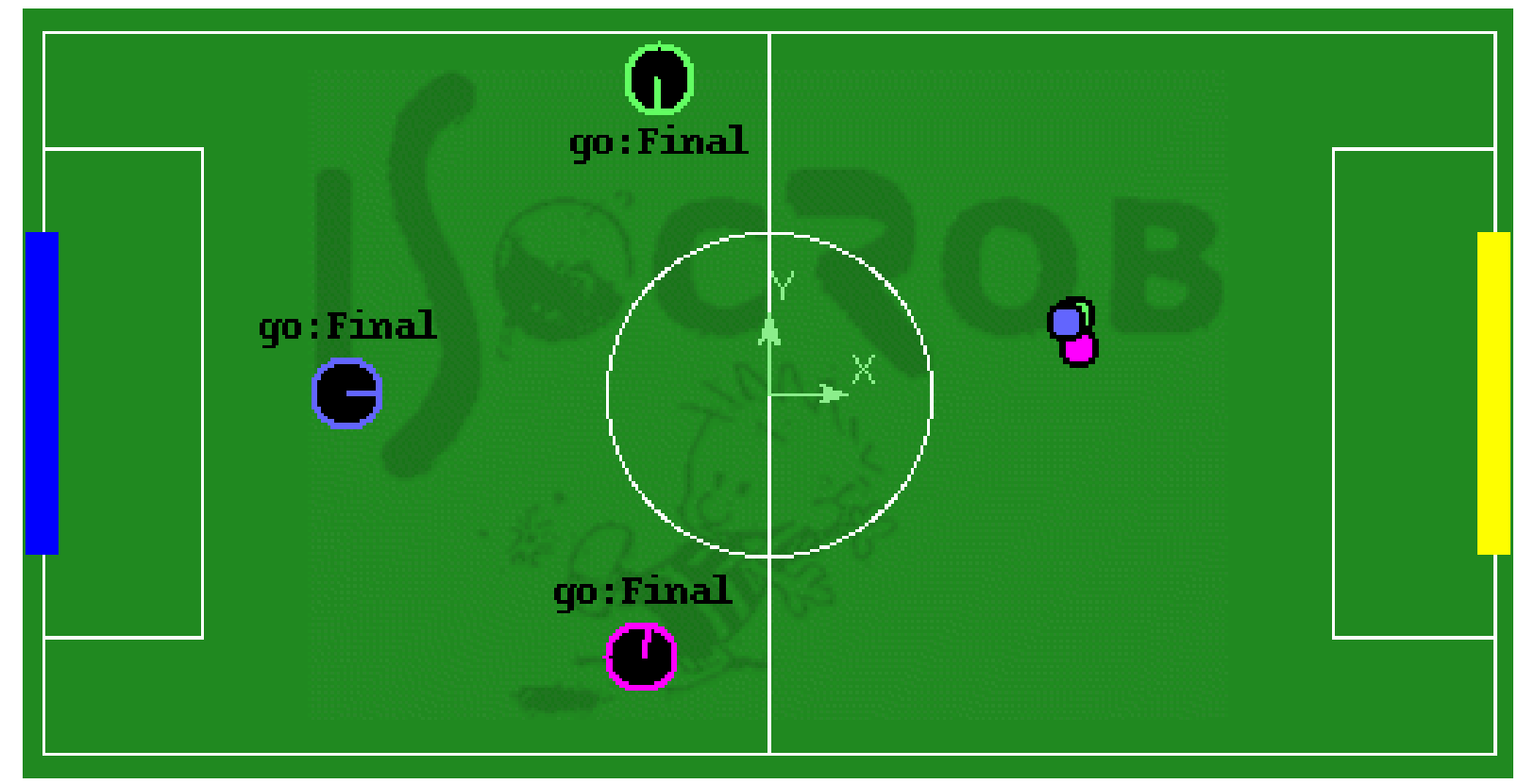




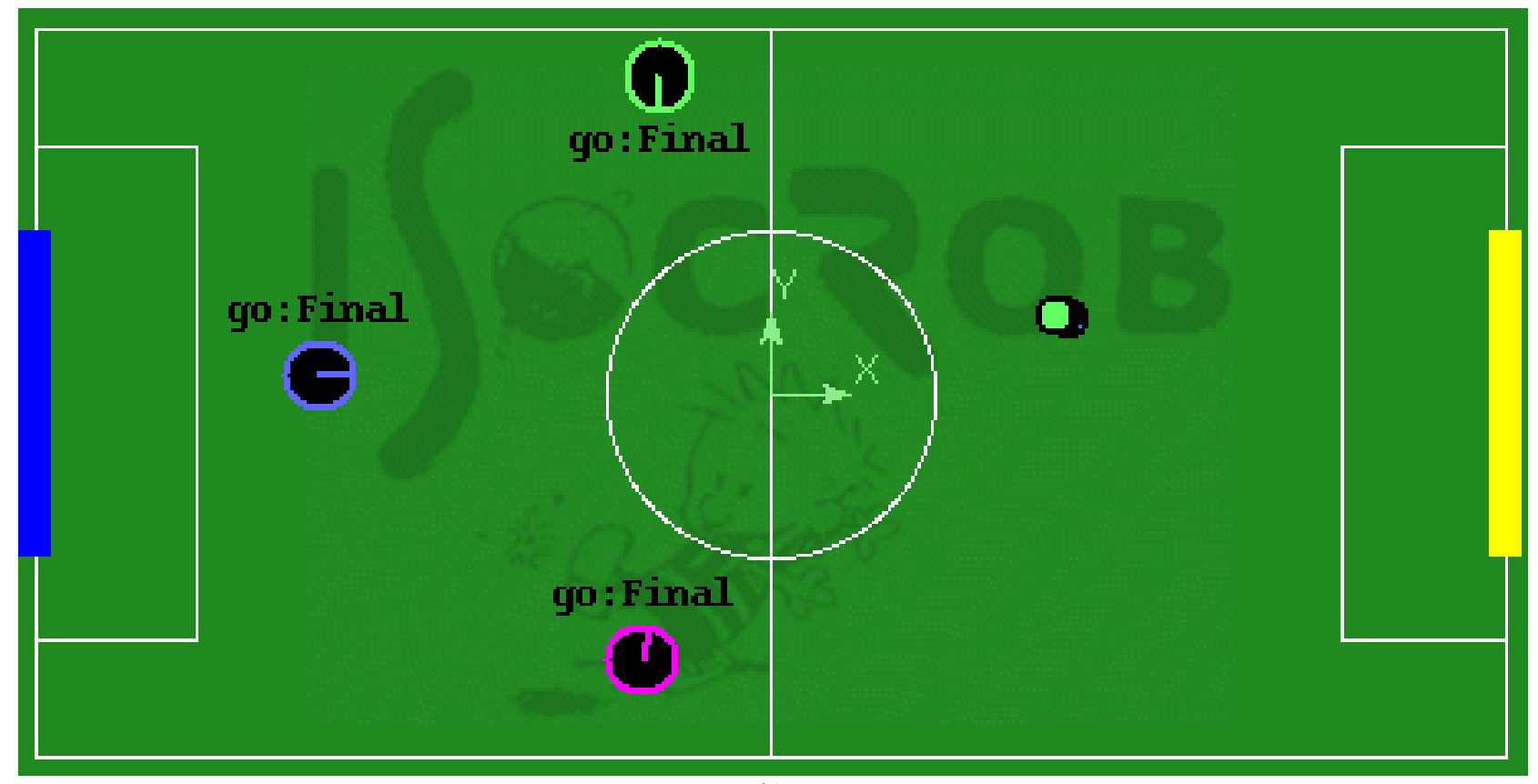

b)

Figure 2. Example of sensor fusion. In the snapshots of the team interface, the larger circles with a mark denoting orientation are robots. The smaller circles represent the ball positions, estimated by each of the robots: a) local (internal to each robot) sensor fusion enabled and global (among team robots) sensor fusion disabled; $b$ ) both local and global sensor fusion enabled.

A topic of current research within the project is the use of sensor fusion for world modeling. The goal is to maintain and update over time information on the relevant objects, such as ball position and velocity, teammates pose and velocity, opponents pose and velocity, or position of the goals with respect to the robot.

Such information is obtained by each robot from the observations of its front and up cameras and then fused among all the team robots (Pinheiro, P. \& Lima, P., 2004), using a Bayesian approach to sensor fusion, as depicted in Fig. 2. Currently this approach is used to provide information on ball position to all the team members, therefore enabling robots that do not see the ball to know where it is, besides improving ball localization reliability. Fusion is not used when two robots disagree (in probabilistic terms) on the ball localization.

\subsection{Vision-Based Self-Localization}

An algorithm that determines the posture of a robot, with respect to a given coordinate system, from the observation of natural landmarks of the soccer field, such as the field lines and goals, as well as from a priori knowledge of the field geometry, has been developed within the SocRob project (Marques, C., \& Lima, P., 2001). The algorithm is a particular implementation of a general method applicable to other well-structured environments, also introduced in (Marques, C., \& Lima, P., 2001).

The landmarks are processed from an image taken by the up cam omni-directional vision system, an image of which is depicted in Fig. 3. The image green-white-green color transitions over a pre-determined number of circles centered with the robot are collected as the set of transition pixels. 


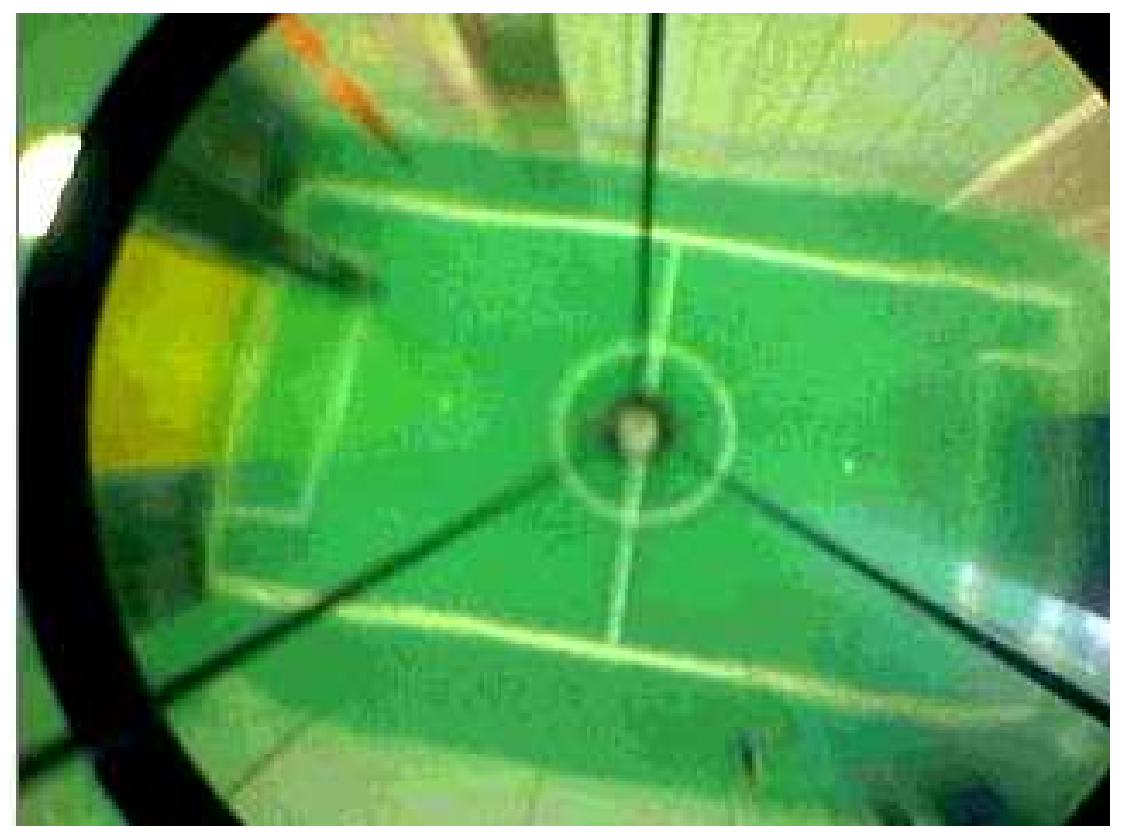

Figure 3. Bird's eye-view of the field obtained by the top catadioptric systems of the robots in Fig. 1

The Hough Transform is applied to the set of transition pixels in a given image, using the polar representation of a line (Gonzalez, R., \& Woods, R., 1992):

$$
\rho=x_{i}^{t} \cdot \cos \phi+y_{i}^{t} \cdot \sin \phi
$$

where $\left(x_{i}^{t}, y_{i}^{t}\right)$ are the image coordinates of transition pixel $\mathrm{p}^{\mathrm{t}}$ and $\rho, \phi$ are the line parameters. The $q$ straight lines $\left(\rho_{1}, \phi_{1}\right), \ldots,\left(\rho_{\mathrm{q}}, \phi_{\mathrm{q}}\right)$ corresponding to the top $\mathrm{q}$ accumulator cells in Hough space are picked and, for all pairs $\left\{\left(\rho_{\mathrm{j}}, \phi_{\mathrm{j}}\right),\left(\rho_{\mathrm{k}}, \phi_{\mathrm{k}}\right), j, k=1, \ldots, q, \mathrm{j} \neq k\right\}$ made out of those $\mathrm{q}$ straight lines the following distances in Hough space are computed:

$$
\begin{aligned}
& \Delta \phi=\left|\phi_{j}-\phi_{k}\right| \\
& \Delta \rho=\left|\rho_{j}-\rho_{k}\right|
\end{aligned}
$$

Note that a small $\Delta \Phi$ denotes almost parallel straight lines, while $\Delta \rho$ is the distance between 2 parallel lines. The $\Delta \Phi$ and $\Delta \rho$ values are subsequently classified by relevance functions which, based on the knowledge of the field geometry, will filter out lines whose relative orientation and/or distances do not match the actual field relative orientation and/or distances. The remaining lines are correlated, in Hough space, with the geometric field model, so as to obtain the robot posture estimate. An additional step must be taken to disambiguate the robot orientation. In the application to soccer robots, the ambiguity is due to the soccer field symmetry. The goal colors are used to remove such ambiguity and to detect situations where the localization values obtained are not trustable.

Currently, an efficiently coded version of the algorithm is used by each of the ISocRob team robots to obtain its self-localization during a game every second. The algorithm runs in parallel with all the other processes and can compute self-localization in about $13 \mathrm{~ms}$ on the average, using Intel IPP library.

The knowledge of each robot localization is useful for individual robot navigation, but it is also used by the robot to share information with its teammates regarding team postures and ball location. 


\subsection{Multi-Sensor Guidance with Obstacle Avoidance}

The ability to navigate at relatively high speeds through an environment cluttered with static and dynamic obstacles is a crucial issue for a mobile robot. Most robotic tasks require a robot to move to target postures adequate to carry out its planned activities. In robotic soccer, relevant activities include facing the opponent goal with the ball in between or covering the team goal by positioning itself between the ball and the goal, while avoiding the field walls and the other (stopped or moving) robots. Also relevant is the capability to move towards a given posture while avoiding obstacles and keeping the ball (also known as dribbling). A guidance control method for non-holonomic (differential drive) vehicles, using odometry, regularly reset by the vision-based self-localization algorithm described before, was first introduced in (Marques, C., and Lima, P., 2002). The vehicle uses a sonar ring for obstacle avoidance.

An alternative guidance method has been introduced in (Damas, B., et al, 2002), consisting of a modified potential fields method for robot navigation, especially suited for differential-drive non-holonomic mobile robots. The potential field is modified so as to enhance the relevance of obstacles in the direction of the robot motion. The relative weight assigned to front and side obstacles can be modified by the adjustment of one physically interpretable parameter. The resulting angular speed and linear acceleration of the robot can be expressed as functions of the linear speed, distance and relative orientation to the obstacles. This formulation enables the assignment of angular and linear velocities for the robot in a natural fashion. Moreover, it leads to an elegant formulation of the constraints on angular speed, linear speed and acceleration, that enable a soccer robot to dribble with the ball, i.e., to move while avoiding obstacles and pushing the ball without losing it, under severe restrictions to ball holding capabilities. It is shown that, under reasonable physical considerations, the angular speed must be less than a non-linear function of the linear speed and acceleration, which reduces to an affine function of the acceleration/speed ratio when a simplified model of the friction forces on the ball is used and the curvature of the robot trajectory is small.

\subsection{Behavior-Based Architectures}

The basic functional architecture of the SocRob team is organized in three levels of team member responsibility, similar to those proposed in (Drogoul, A., and Collinot, A., 1998): individual, which is responsible for all functionalities that involve only one robot; relational, which is responsible for the relationships between the robot and its teammates; and organizational, which is responsible for the strategic decisions that involve the team as a whole. Behaviours are classified according to this division: we consider organizational, relational and individual behaviours.

Since behaviours are externally displayed and emerge from the application of certain operators, the functional architecture can also be viewed from an operator standpoint, with three levels of decision:

- Team Organization Level, where, based on the current world model, a strategy (i.e., what to do) is established, including a goal for the team. This level considers issues such as modelling the opponent behaviour to plan a new strategy. Strategies may simply consist of enabling a given subset of the operators at each robot, in result of role assignments to each team member. In robotic soccer, basic roles can be Goalkeeper, Defender, Attacker and Full Player (both defender and attacker). Only the captain robot will have the 
organization level enabled. Should the captain "die", the next robot in a pre-specified list will have its organization level enabled and become the captain.

- Behaviour Coordination Level, where switching among operators, both individual and relational, occurs so as to coordinate behaviour execution, at each robot and among the team robots, towards achieving the team goal, effectively establishing the team tactics (i.e., how to do it). Both a finite state automaton or a rule-based system were used to implement this level, but other alternative representations are possible, such as Petri nets.

- Behaviour Execution Level, where primitive tasks run and where they interface the sensors, through the blackboard, and the actuators, through the navigation functions at each robot. Primitive tasks are linked to each other to implement an operator. Currently, every operator (representing a given behaviour) is implemented as a finite state automaton whose states are the primitive tasks and transitions are associated to logical conditions on events that are detected by the system. Behaviours can be individual, if their corresponding operators run in one robot only, or relational, if two or more robots are running operators that are coordinated through commitments and synchronisation messages to achieve a common goal (e.g., to pass a ball, to avoid moving simultaneously towards a ball, to cover a field region while the teammate advances in the field through role exchanges). Any team member may have relational operators available. Each operator has a pre-conditions set and, when this set is satisfied, establishes communications with the relational operator(s) of designated teammates, asking them to start a negotiation process which may end up in a coordinated action among this temporary sub-team. As a result, a relational behaviour is displayed.

The software architecture is the practical implementation of the functional architecture, which could be done in any programming language and using different software technologies. In the SocRob project, the software architecture was defined based on three essential concepts: micro-agents ( $\mu$ A for short), blackboard and plugins.

Inspired by the idea of Society of Agents, proposed by Minsky (Minsky, M., 1988), each functional module of the SocRob architecture was implemented by a separate process, using the parallel programming technology of threads. In this context a functional module is named $\mu \mathrm{A}$. In the current implementation of the SocRob architecture there are nine different threads, but only the four most important ones are mentioned here: $\mu \mathrm{A}$ Vision, responsible for processing the data acquired from the cameras, $\mu \mathrm{A}$ Fusion, which fuses information concerning the same object from different sensors, $\mu \mathrm{A}$ Machine, responsible for deciding which behavior should the robot display, and $\mu \mathrm{A}$ Control, responsible for the execution of the corresponding operator.

The concept of threads was chosen to improve module performance and simplify the information passing among the threads. This was accomplished by the blackboard concept (memory space shared by several threads), further sophisticated here by the development of a distributed blackboard, in what information availability is concerned. Instead of being centralized in one agent, the information is distributed among all team members and communicated when needed.

As mentioned before, the decision making involved for each agent is twofold: which behavior should be displayed, and how the operator which displays such behavior is executed. This separation between behavior decision and operator execution allows the $\mu$ A Machine, the one responsible for behavior decision, to work with abstract definitions of behaviors, and choose among them without knowing details about their execution. So, new operators could be easily added and removed without affecting the existing ones, and 
these can also be easily replaced by others with the simple restriction of maintaining the name. This was accomplished using the concept of plugin, in the sense that each new operator is added to the software architecture as a plugin, and therefore the $\mu \mathrm{A}$ Control can be seen as a multiplexer of plugins. Examples of already implemented operators are: dribble, score, go, standby, to name but a few. The same idea of plugins was also used for the $\mu \mathrm{A}$ Vision, as each particular functionality related to vision data is defined as a different plugin, and multiplexed by the $\mu \mathrm{A}$ Vision (e.g., a plugin for the front camera, a plugin for the up camera, a plugin for the self-localization algorithm, etc.).

The individual operators have been implemented as state machines, where the states represent primitive tasks, while the arcs between states (if any) are traversed upon the validation of given logical conditions over events (e.g., see ball, distance < x). The relational operator state machines could be defined similarly, but events include synchronization signals between the state machines running in the sub-team robots.

However, the way the functional architecture was conceptualized allows the implementation of these operators and the switching among them using different approaches, as for example AI production systems. So, in order to have a more abstract way to deal with operator/behaviour switching, the $\square \mathrm{A}$ Machine has been implemented using a distributed decision-making architecture supported on a logical approach to modeling dynamical systems (Reiter, 2001), based on situation calculus, a first order logic dialect.

This architecture includes two main modules (see Fig. 4):

- a basic logic decision unit, and

- an advanced logic decision unit.

Both run in parallel; the former intends to quickly suggest, using simple logical decision rules, the next operator/behaviour to be executed, whereas the latter uses more sophisticated reasoning tools (situation calculus) capable of planning, learning and decision-making, both for individual and cooperative (teamwork) situations. This configures an hybrid architecture where the basic (reactive) unit only controls the robot if the advanced (deliberative) unit takes too long to make a decision, assuming a situation urgency evaluation. A partial implementation of this architecture, the basic logic decision unit, was already performed using Prolog (Arroz, M., et al, 2004). Its modeling convenience allowed the quick development of different roles for field players (Attacker, Defender, Full-Player), as well as dynamic role change between field players (defenders switch with attackers, depending on who is in a better position to get the ball).

The advanced (deliberative) unit, Advanced Logic Based Unit, has been developed using an action programming language called Golog Golog (Levesque, H., et al, 1997) and it is based on situation calculus. This unit is responsible to determine plans (sequences of operators/behaviours) that allow the team to achieve something (like scoring on the opposite goal). Situation calculus is an extension to first-order logic, specially suited to handle dynamic worlds. The changes in the world are the results of actions that have preconditions and effects.

Our objective is to develop a tool capable of planning and performing task control execution in a distributed environment. To do so we assume that: the agents (robots) can generate, change and execute plans; a plan can be generated, and executed by one or more agents; decisions over the generated plans are based on hypotheses, i.e., assumptions over future states that cannot be guaranteed; and the agents have the capacity to communicate among them, and share information about plans or environment states. 


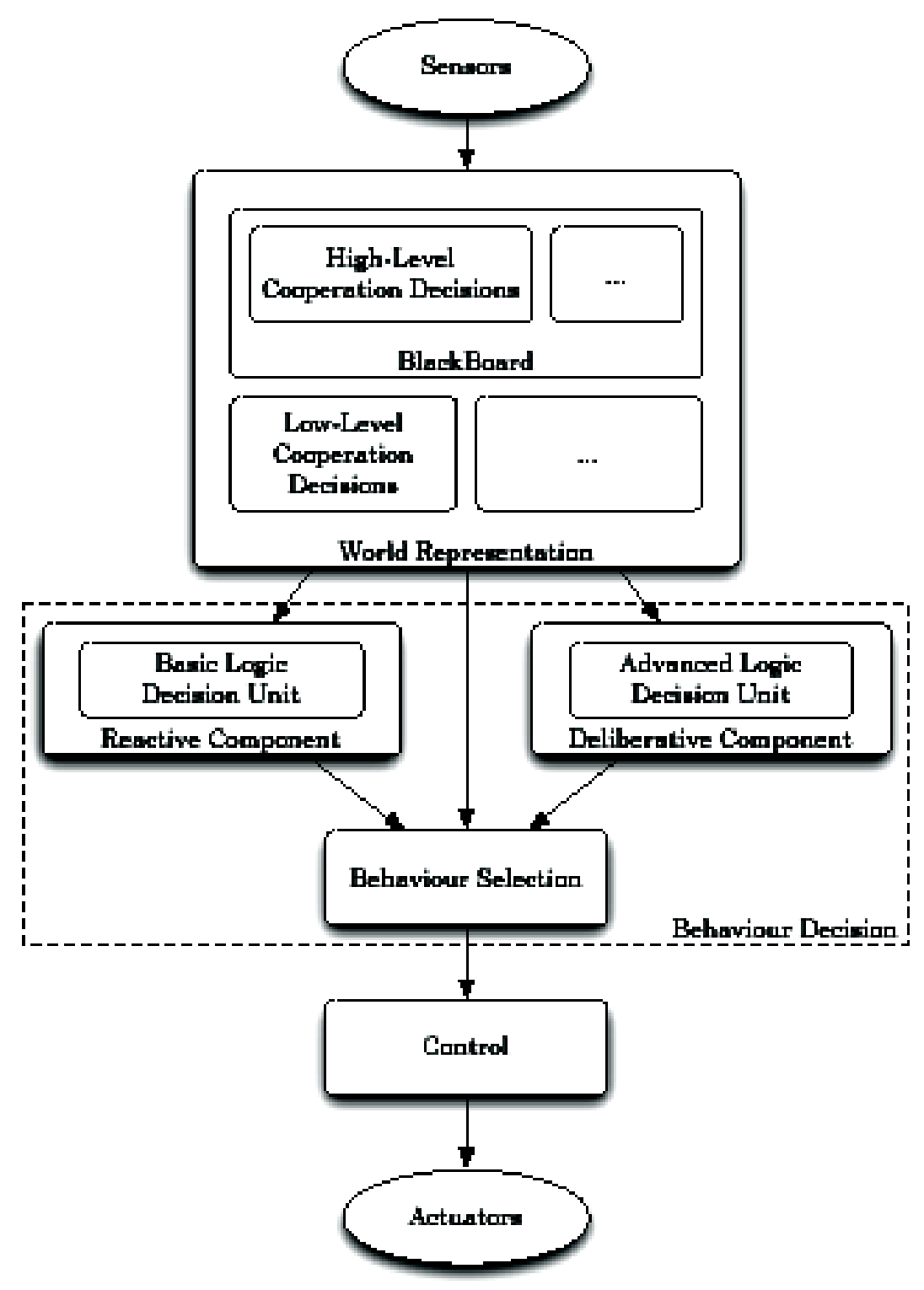

Figure 4. Hybrid architecture of the $\mu$ A machine

\subsection{Relational Operators}

Another recent topic in the project research is the design and implementation of relational behaviors, where teamwork between two or more robots is required to perform a certain task, like a ball pass (Vecht, B., \& Lima, P., 2004). These behaviors have a general formulation based on Joint Commitment Theory (Cohen, P. R., \& Levesque, H. J., 1991), and use the navigation methods already developed in the project.

Currently, the robots are capable of committing to a relational pass behavior where one of the robots is the kicker and the other the receiver. Should any of the robots end the commitment (e.g., due to a failure to achieve the behaviour goal or after succeeding in it), it inform the other(s) robot(s) involved in the relational operator, so that they can take the appropriate actions to end the commitment and switch to another operator.

One cooperation mechanism, implemented in 2000, consists of avoiding that two or more robots from the same team attempt to get the ball. A relational operator was developed to determine which robot should go to the ball and which one(s) should not. In the current implementation, each robot that sees the ball and wants to go for it uses a heuristic 
function to determine a fitness value. This heuristic penalizes robots that are far from the ball, are between the ball and the opposite goal and need to perform a angular correction to center the ball with its kicking device. Each robot broadcasts its own heuristic value, and the robot with the smallest value is allowed to go for the ball whereas the others execute a Standby behavior.

\subsection{Task Planning and Allocation}

Though not tested yet in real robots, formal work on Stochastic Discrete-Event Systems modeling of a multi-robot team has been recently carried out within the project with interesting results (Damas, B., \& Lima, P., 2004).

The environment space and each player (opponent and teammate) actions are discretized and modeled by a Finite State Automaton (FSA) representing a 2 vs 2 players game. Then, all FSA are composed to obtain the complete model of a team situated in its environment and playing an adversarial game. Controllable (e.g., shoot_p1, stop_p2) and Uncontrollable (e.g., lost_ball, see_ball) events (i.e., our robots actions) are identified and exponential distributions are assigned to their inter-event times. Dynamic programming is applied to the optimal selection of the controllable events, with the goal of minimizing the cost function

$$
\min _{\pi}\left[\int_{0}^{\infty} C[X(t), u(t)] d t\right]
$$

where $\pi$ is a policy, $X(t)$ the game state at time $t$, and $u(t)$ is a controllable event, with the cost of unmarked states equal to 1 , and all the other states have zero cost. If the only marked states are those where a goal is scored for our team, and there are no transitions from marked to unmarked states, this method obtains the minimum (in a stochastic sense) time to score a goal for our team, constrained by the opponent actions and the uncertainty of our own actions. Some of the chosen actions result in cooperation between the two robots of the team.

\section{Problems to be Addressed}

Naturally, several interesting problems remain to be tackled and solved within the project research. We will only mention the currently most important ones.

Behavior Modeling: A consistent model for individual and relational behaviors, or more precisely, for the operators implementing them, is required to provide a systematic methodology for behavior synthesis and analysis. FSA have been used for this purpose up to now. They have the advantage of the availability of several tools for analysis and synthesis in the literature (Cassandras, C. G., \& Lafortune, S, 1999), but suffer from limited modeling capabilities. Petri nets (Cassandras, C. G., \& Lafortune, S, 1999) extend the modeling capabilities of FSA and provide a more convenient modeling methodology starting from the identification of the system components and events. A wide range of analysis (e.g., concerning boundedness, liveness, stochastic and deterministic time) and synthesis (e.g., concerning admissible marked languages) tools is also available, and the non-decidability of some analysis problems can be overcome with no significant expenses. Furthermore, modularity and system design can be achieved by interconnecting several 
sub-systems, each modeled as a Petri net. This is particularly convenient to model relational behaviors, where more than one teammate is involved. So, Petri nets are being investigated as an alternative tool for behavior modeling. Behavior switching can also be modeled as discrete-event systems supervision, for which there are results available regarding FSA and Petri nets. Production systems also have modeling characteristics that make them suitable for this purpose. However, further work must be done to study its design and analysis properties.

Distributed Planning: The available behaviors among which switching is possible are currently designed "by hand". However, a more appropriate approach would be to develop a planner capable of periodically (or when invoked) analyzing the world state and providing a new set of individual and relational behaviors appropriate for the current conditions. A suitable approach should be the continuous interleaving of plan generation and execution. Task allocation among the team robots and distributed world modeling are relevant issues to be further investigated under this topic. Another relevant issue under investigation is the translation into behaviour models (e.g., FSA, Petri nets) of logical specifications for a mission to be carried out by a team of robots.

Cooperative Learning: One possible way of designing plans which continuously adapt to new situations and are fine tuned to the actual surrounding environment is to use reinforcement learning (RL) algorithms, especially those which guarantee convergence properties (Sutton, R., and Barto, A., 1998). However, learning is usually slow. An envisaged approach that overcomes this problem is to provide plans with alternative paths among which the RL algorithms can learn to switch over time. Cooperative learning arises when a robot takes its decisions from information learned and provided to it by its teammates.

Control as a Game: Modern views of control state the control problem as a game against an adversary (i.e., the disturbances). In the particular case of soccer, there is an actual opponent whose modeled behavior, once estimated (e.g., using Hidden Markov Models), can be used as information for game-playing algorithms, as part of the planning process.

\section{Conclusions}

This paper described the SocRob project (on the development of methodologies for analysis, design and implementation of multi-robot cooperative systems), its objectives, past, current and intended future work. One interesting feature of the project is that it enables different approaches to the solution of the problem at hand. This naturally motivates competing research approaches, as well as research on analysis methods to compare the different results.

Furthermore, the project fosters education in AI and Robotics related topics, because so many issues must be solved to handle the overall problem. Students from different levels (undergraduate, graduate, post-doctorate) can get involved at different difficulty levels and accomplish project sub-goals. They also learn how to accomplish teamwork under hard time deadlines. The SocRob project has involved so far 20 undergraduate and 8 graduate (5 MSc and $3 \mathrm{PhD}$ ) students, besides 2 doctorates who have been supervising the project. All these students have participated regularly in RoboCup - The World Cup of Soccer Robots, since 1998. We believe that RoboCup is a very attractive long-term scientific challenge that brings together people from several different scientific fields in an exciting fusion of research, education and science promotion which are actually the driving forces of our project too. 
Some of the methodologies developed within the project, namely its software and functional architectures, have been applied meanwhile to other projects, such as an European Space Agency project on Formation Guidance and Navigation of Distributed Spacecraft, and a Cooperative Navigation for Rescue Robots project currently underway at ISR/IST. The project team is now developing new robots, in the framework of a national research project, in partnership with two Portuguese small companies. These new robots are omnidirectional, with a new modular construction, so that it will be easily modified, e.g., the up camera module can switch between a catadioptric system and a stereo image system.

The new robots will also incorporate a controlled kicker mechanism, so that one can choose the kicking force, using an electromechanical solution with a DC motor pulling a spring and an infrared sensor to measure the pulled distance, both coupled to the kicking device. In order to make new and more complex behaviors and for ball handling, there is a ball reception mechanism, that will allow the implementation of ball passes behaviors. Two new sensors will be used: a rate-gyro for angular velocity measurements, and an optical mouse to track the robot position in the field. Both will provide data to be fused with odometry and vision-based self-localization, so as to improve navigation.

\section{References}

Balch, T. and Parker, L. (eds), Robot Teams: From Diversity to Polymorphism, AK Peters, 2002.

Canudas de Wit, C. and Siciliano B., and Bastin G. (Eds) (1996), Theory of Robot Control, CCE Series, Kluwer

Cassandras, C. G. and Lafortune, S. (1999), Introduction to Discrete Event Systems, Kluwer Academic Publishers

Cohen, P. R., and Levesque, H. J. (1991), "Teamwork". Nous, Vol 35, pp. 487-512

Damas, B. and Lima P. and Custódio (2002), "A Modified Potential Fields Method for Robot Navigation Applied to Dribbling in Robotic Soccer", Proceedings of RoboCup2002 Symposium, Fukuoka, Japan

Damas, B., and Lima, P., (2004), "Stochastic Discrete Event Model of a Multi-Robot Team Playing an Adversarial Game", 5th IFAC/EURON Symposium on Intelligent Autonomous Vehicles - IAV2004, Lisboa, Portugal

Decker, K. S., and Lesser, V. R. (1995), "Designing a Family of Coordination Algorithms", Technical Report No. 94-14, Department of Computer Science, University of Massachussets, Amherst, MA01003

desJardins, M. E., and Durfee, E. H., and Ortiz Jr, C. L., and Wolverton, M. J. (1999), “A Survey of Research in Distributed, Continual Planning", AI Magazine, Winter, pp. 13-22

Drogoul, A., and Collinot, A. (1998), "Applying an Agent-Oriented Methodology to the Design of Artificial Organizations: A Case Study in Robotic Soccer", Autonomous Agents and Multi-Agent Systems Journal, Vol. 1, pp. 113-129

Ferber, J. (1999), Multi-Agent Systems: An Introduction to Distributed Artificial Intelligence, Addison-Wesley

Gonzalez, R., and Woods, R. (1992), Digital Image Processing, Addison-Wesley

Levesque, H., and Reiter, R., and Lesprance, Y., and Lin, F., and Scherl, R. (1997), “Golog: A Logic Programming Language for Dynamics Domains". Journal of Logic Programming 
Marques, C., and Lima, P. (2001), "A Localization Method for a Soccer Robot Using a Vision-Based Omni-Directional Sensor", RoboCup-2000: Robot Soccer World Cup IV, P. Stone, T. Balch, G. Kraetzschmar (Eds.), Springer-Verlag, Berlin

Marques, C., and Lima, P. (2002), "Multi-sensor Navigation for Soccer Robots", RoboCup2001: Robot Soccer World Cup V, A. Birk, S. Coradeschi, S. Tadokoro (Eds.), Springer-Verlag, Berlin

Minsky, M. (1988), The Society of Mind, Touchstone Publishers

Pinheiro, P., and Lima, P. (2004), "Bayesian Sensor Fusion for Cooperative Object Localization and World Modeling", 8th Conference on Intelligent Autonomous Systems (IAS-8), Amsterdam, The Netherlands, May 2004.

Pires, V., Arroz, M., and Custódio, L. (2004), “Logic Based Hybrid Decision System for a Multi-robot Team", Proceedings of the 8th Conference on Intelligent Autonomous Systems (IAS-8), Amsterdam, The Netherlands.

Pires, V., Arroz, M., Lima, P., Ribeiro, M. I., and Custódio (2004), L., “Distributed Deliberative Decision System for a Multi-Robot Team", Proceedings of the ROBOTICA 2004 Symposium, Porto, Portugal, April 2004.

Reiter, R. (2001), Knowledge in Action. MIT Press

Sutton, R., and Barto, A. (1998), Reinforcement Learning, MIT Press, Cambridge, MA

Vecht, B., Lima, P., 2004, "Formulation and Implementation of Relational Behaviors for Multi-Robot Cooperative Systems". Proceedings of RoboCup 2004 Symposium, Lisbon, Portugal 


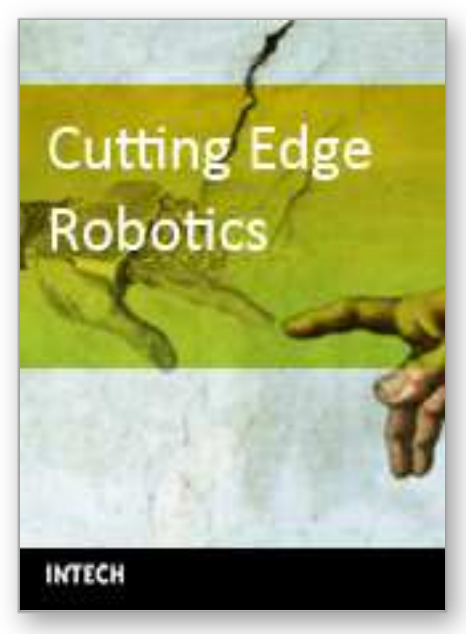

\section{Cutting Edge Robotics}

Edited by Vedran Kordic, Aleksandar Lazinica and Munir Merdan

ISBN 3-86611-038-3

Hard cover, 784 pages

Publisher Pro Literatur Verlag, Germany

Published online 01, July, 2005

Published in print edition July, 2005

This book is the result of inspirations and contributions from many researchers worldwide. It presents a collection of wide range research results of robotics scientific community. Various aspects of current research in robotics area are explored and discussed. The book begins with researches in robot modelling \& design, in which different approaches in kinematical, dynamical and other design issues of mobile robots are discussed. Second chapter deals with various sensor systems, but the major part of the chapter is devoted to robotic vision systems. Chapter III is devoted to robot navigation and presents different navigation architectures. The chapter IV is devoted to research on adaptive and learning systems in mobile robots area. The chapter V speaks about different application areas of multi-robot systems. Other emerging field is discussed in chapter VI - the human- robot interaction. Chapter VII gives a great tutorial on legged robot systems and one research overview on design of a humanoid robot. The different examples of service robots are showed in chapter VIII. Chapter IX is oriented to industrial robots, i.e. robot manipulators. Different mechatronic systems oriented on robotics are explored in the last chapter of the book.

\section{How to reference}

In order to correctly reference this scholarly work, feel free to copy and paste the following:

Pedro U. Lima and Luis M. M. Custodio (2005). The SocRob Project: Soccer Robots or Society of Robots, Cutting Edge Robotics, Vedran Kordic, Aleksandar Lazinica and Munir Merdan (Ed.), ISBN: 3-86611-038-3, InTech, Available from:

http://www.intechopen.com/books/cutting_edge_robotics/the_socrob_project_soccer_robots_or_society_of_r obots

\section{INTECH}

open science | open minds

\author{
InTech Europe \\ University Campus STeP Ri \\ Slavka Krautzeka 83/A \\ 51000 Rijeka, Croatia \\ Phone: +385 (51) 770447 \\ Fax: +385 (51) 686166 \\ www.intechopen.com
}

\author{
InTech China \\ Unit 405, Office Block, Hotel Equatorial Shanghai \\ No.65, Yan An Road (West), Shanghai, 200040, China \\ 中国上海市延安西路65号上海国际贵都大饭店办公楼 405 单元 \\ Phone: $+86-21-62489820$ \\ Fax: +86-21-62489821
}


(C) 2005 The Author(s). Licensee IntechOpen. This chapter is distributed under the terms of the Creative Commons Attribution-NonCommercial-ShareAlike-3.0 License, which permits use, distribution and reproduction for non-commercial purposes, provided the original is properly cited and derivative works building on this content are distributed under the same license. 